\title{
Existe diferença na contratilidade da musculatura do assoalho pélvico feminino em diversas posiçōes?
}

Is there any difference between female pelvic floor muscle contractility in different positions?

\author{
Mariana Tirolli Rett ${ }^{1}$, José Antonio Simões ${ }^{2}$, Viviane Herrmann ${ }^{3}$, \\ Andréa de Andrade Marques ${ }^{4}$, Sirlei Siani Morais ${ }^{5}$
}

\section{RESUMO}

Objetivo: avaliar e comparar resultados da eletromiografia de superfície do assoalho pélvico feminino em diversas posições (decúbito dorsal, na posição sentada e ortostática). Métodos: foram avaliadas 26 mulheres submetidas a um protocolo de exercícios para o fortalecimento do assoalho pélvico como tratamento da incontinência urinária de esforço por hipermobilidade do colo vesical. Utilizou-se sensor intravaginal conectado ao equipamento Myotrac $3 \mathrm{G}^{\mathrm{TM}}$ e a avaliação consistia em: 60 segundos iniciais de repouso, cinco contrações fásicas, uma contração tônica de 10 segundos e outra de 20 segundos. As amplitudes destas contrações foram obtidas pela diferença entre a amplitude final da contração menos a amplitude de repouso $(\mathrm{em} \mu \mathrm{V})$ e foram comparadas com o uso do teste de Wilcoxon para amostras pareadas $(\mathrm{p}<0,05)$. Resultados: as amplitudes das contrações foram maiores em decúbito dorsal, decrescendo sucessivamente nas posições sentada e ortostática. Em decúbito dorsal, as medianas das contrações fásicas e tônicas de $10 \mathrm{~s} \mathrm{e} 20 \mathrm{~s}$ foram respectivamente 23,5 (5-73), 18,0 (3$58)$ e 17,0 (2-48). Na posição sentada foram 20,0 (2-69), 16,0 (0-58) e 15,5 (1-48), e na posição ortostática 16,5 (3-67), 12,5 (254) e 13,5 (2-41). Quando se comparou a posição ortostática, com o decúbito dorsal, observou-se diferença significativa em todas as contrações $(p<0,001, p<0,001$ e p =0,003). Resultados semelhantes também foram encontrados comparando-se a posição ortostática com a posição sentada. Todavia, entre o decúbito dorsal e a posição sentada, não foi observada diferença significativa. Conclusão: as amplitudes de todas as contrações do assoalho pélvico feminino foram inferiores na posição ortostática, sugerindo que o fortalecimento muscular deve ser intensificado nesta posição.

PALAVRAS-CHAVE: Incontinência urinária de esforço; Assoalho pélvico; Eletromiografia

\section{ABSTRACT}

Purpose: to evaluate and compare results of female pelvic floor surface electromyography in different positions: lying, sitting and standing. Methods: twenty-six women with the diagnosis of stress urinary incontinence treated with a protocol of exercises to strengthen the pelvic floor muscle were evaluated. Pelvic floor surface electromyography was performed with an intravaginal sensor connected to Myotrac $3 \mathrm{G}^{\mathrm{TM}}$ equipment, as follows: initial rest of $60 \mathrm{~s}$, five phasic contractions, one 10 -s tonic contraction and one 20-s tonic contraction. The amplitudes were obtained from the difference between the final contraction amplitude and the amplitude at rest (in $\mu \mathrm{V}$ ). Wilcoxon test was applied for nonparametric data ( $\mathrm{p}$ value $<0.05$ ). Results: the amplitudes of contractions were higher in the lying position, decreasing in the sitting and standing positions. In the lying position, the median values of phasic and tonic contractions were 23.5 (5-73), 18.0 (3-58) and 17.0 (2-48), respectively. In the sitting position, they were 20.0 (2-69), 16.0 (0-58) and 15.5 (1-48). In the standing position they were 16.5 (3-67), 12.5 (2-54) and 13.5 (2-41). All amplitude values were significantly lower in the standing position compared to the lying position $(\mathrm{p}<0.001, \mathrm{p}<0.001$ and $\mathrm{p}=0.003)$. Similar results were also found in comparison to the sitting position. However, there was no significant difference between the lying and the sitting positions. Conclusion: all female pelvic floor contraction amplitudes were lower in the standing position, suggesting that the muscle strength should be intensified in that position.

KEYWORDS: Stress urinary incontinence; Pelvic floor; Electromyography

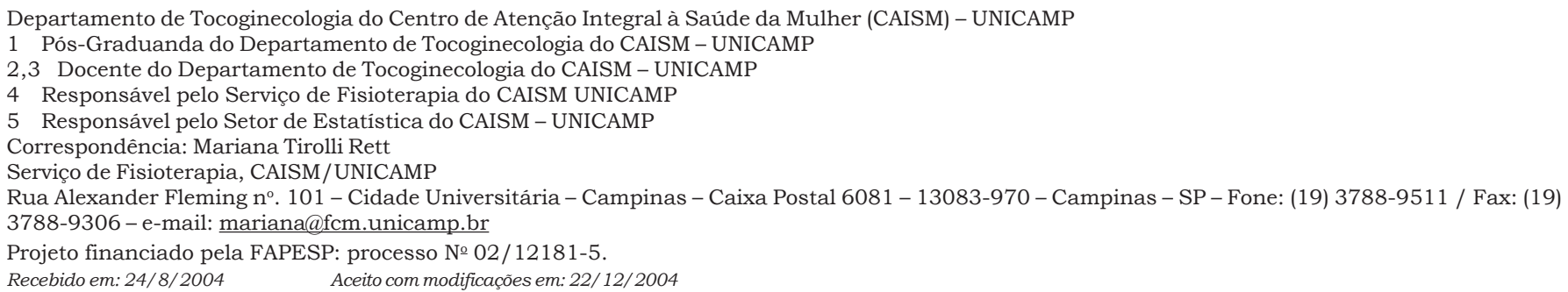




\section{Introdução}

A avaliação da função e da força muscular do assoalho pélvico tem papel decisivo no tratamento fisioterápico da incontinência urinária, principalmente para a incontinência urinária de esforço (IUE) ${ }^{1-3}$. Além disso, representa importante ferramenta para monitorização dos resultados clínicos e, muitas vezes, serve como forma de aprendizado e motivação para a paciente ${ }^{3}$. O assoalho pélvico, importante estrutura no suporte uretral e no mecanismo de continência, é tradicionalmente avaliado por meio da palpação e da perineometria ${ }^{1-4}$. Como os músculos do assoalho pélvico são compostos por fibras musculares estriadas esqueléticas do tipo I (contração lenta ou tônica) e tipo II (contração rápida ou fásica) ${ }^{5}$, também podem ter sua atividade avaliada pela eletromiografia de superficie (sEMG) $)^{1,3,6-8}$.

A sEMG registra os potenciais elétricos gerados pela despolarização das fibras musculares em repouso e durante a contração voluntária, podendo ser considerada medida indireta de força muscular. Além de avaliar a capacidade de contração, a sEMG registra a sua amplitude em microvolts $(\mu \mathrm{V})$, sendo que os valores registrados em repouso caracterizam o tônus basal do músculo ${ }^{8}$. Durante a contração muscular, a sEMG oferece informações sobre as fibras fásicas, que produzem contrações de máxima amplitude e curta duração, e sobre as fibras tônicas, que produzem contrações de menor amplitude e maior tempo de duração ${ }^{8}$.

$\mathrm{Na}$ maioria das vezes, esta forma de avaliação do assoalho pélvico é realizada em decúbito dorsal. Isso se deve à maior comodidade do terapeuta e da própria paciente, ou ainda pela facilidade de padronização que esta posição oferece. Todavia, a avaliação em decúbito dorsal acaba representando somente um dos comportamentos que o assoalho pélvico pode assumir ${ }^{3}$. Sabe-se que as queixas de perda urinária em mulheres com IUE são dependentes dos esforços físicos e geralmente exacerbam-se na posição ortostática, que é a mais assumida durante as atividades diárias e na qual há forte ação da gravidade sobre o assoalho pélvico ${ }^{3}$. Estudos para investigar a influência da postura no posicionamento do colo vesical mostraram que há maior mobilidade da uretra proximal e do colo vesical na posição ortostática do que em decúbito dorsal, sendo esta uma possivel explicação para a maior intensidade das queixas urinárias nesta posição ${ }^{4}$. Por isso, alguns exames propedêuticos, como o estudo urodinâmico, são realizados na posição ortostática, a fim de reproduzir a perda urinária de maneira mais fidedigna com a realidade ${ }^{9}$.

Torna-se, assim, importante investigar o comportamento dos músculos do assoalho pélvico em diferentes posições, particularmente na posição ortostática. O objetivo deste trabalho foi avaliar e comparar a eletromiografia de superficie (sEMG) do assoalho pélvico feminino em diversas posições (decúbito dorsal, na posição sentada e na ortostática).

\section{Pacientes e Métodos}

Este estudo foi desenvolvido no Serviço de Fisioterapia do Centro de Atenção Integral à Saúde da Mulher (CAISM) da Universidade Estadual de Campinas (UNICAMP). O projeto foi aprovado pelo Comitê de Ética em Pesquisa da Faculdade de Ciências Médicas da UNICAMP e todas as participantes assinaram o termo de consentimento livre e esclarecido. Foram incluídas 26 mulheres que haviam sido submetidas a um protocolo de tratamento fisioterápico para IUE por hipermobilidade do colo vesical, no período de outubro de 2003 a junho de 2004. O protocolo consistia em exercícios para o fortalecimento do assoalho pélvico, realizados em decúbito dorsal, na posição sentada e ortostática. As mulheres foram submetidas a 12 sessões de tratamento, realizadas duas vezes por semana, durante 6 semanas consecutivas.

Antes de iniciar o tratamento, todas as mulheres foram submetidas a estudo urodinâmico para excluir IUE por deficiência esfincteriana intrínseca da uretra, hiperatividade do detrusor e obstrução infravesical. Os outros critérios de exclusão foram: ser menopausada, apresentar cistocele maior que grau II e ter sido previamente submetida à correção cirúrgica de IUE.

O tempo médio da queixa de IUE variou de dois a dez anos, com média de 5,4 anos $(\mathrm{DP}=3,5)$. A idade das mulheres variou de 31 a 52 anos, com média de 42,6 anos ( $\mathrm{DP}=5,4)$, e o índice de massa corpórea variou de 21,2 a $34,7 \mathrm{~kg} / \mathrm{m}^{2}$, com média de $24,5 \mathrm{~kg} / \mathrm{m}^{2}(\mathrm{DP}=0,7)$. Em relação aos antecedentes obstétricos, apenas uma mulher era nulipara, 14 tiveram de um a três partos e 11 tiveram quatro ou mais partos. Quanto à via de parto, 19 mulheres tiveram no mínimo um parto vaginal e sete tiveram apenas cesárea.

As avaliações eletromiográficas do assoalho pélvico foram realizadas em decúbito dorsal, nas posições sentada e ortostática com o uso de sensor intravaginal conectado ao equipamento Myotrac 3G (Thought Technology, Montreal, Canadá). Estas avaliações consistiam em: 60 segundos iniciais de repouso, cinco contrações fásicas, uma contração 
tônica de 10 segundos e uma contração tônica de 20 segundos $^{7,10,11}$. O software do equipamento registrava as amplitudes destas contrações em microvolts $(\mu \mathrm{V})$, obtidas pela diferença entre a amplitude final da contração e a amplitude de repouso. As medianas das amplitudes das contrações nas diferentes posições foram comparadas com o uso do teste de Wilcoxon para amostras pareadas, com nível de significância de 0,05.

\section{Resultados}

Como pode ser observado na Tabela 1 , em decúbito dorsal as medianas das amplitudes das contrações fásicas e tônicas de $10 \mathrm{~s}$ e $20 \mathrm{~s}$ foram respectivamente 23,5 (5-73), 18,0 (3-58) e 17,0 (2-48). $\mathrm{Na}$ posição sentada, estas medianas das mesmas contrações foram 20,0 (2-69), 16,0 (0-58) e 15,5 (1-
48). Já na posição ortostática, as medianas das amplitudes foram 16,5 (3-67), 12,5 (2-54) e 13,5 (2-41).

Quando se comparou o decúbito dorsal com a posição ortostática, houve diferença significativa nas amplitudes de todos os tipos de contrações $(\mathrm{p}<0,001, \mathrm{p}<0,001$ e $\mathrm{p}=0,003)$. Da mesma forma, todas estas amplitudes de contrações também foram significativamente menores na posição ortostática do que na posição sentada $(p=0,027$, $\mathrm{p}=0,027$ e $\mathrm{p}=0,019$ ). Por outro lado, entre o decúbito dorsal e a posição sentada, nenhuma diferença significativa foi observada, demonstrando que as mulheres foram capazes de gerar contrações de amplitudes semelhantes nestas duas posições $(p=0,172, p=0,098$ e $p=0,204)$.

Em relação às medianas das amplitudes de repouso, foi observada diferença significativa somente entre as posições de decúbito dorsal $(3,0$ $(0-10)$ e ortostática $(4,0(1-11), p=0,010$.

Tabela 1 - Comparação das amplitudes de contração avaliadas pela eletromiografia de superfície (sEMG) do assoalho pélvico em decúbito dorsal, nas posições sentada e ortostática ( $\mathrm{n}$ = 26).

\begin{tabular}{|c|c|c|c|c|c|c|}
\hline \multirow{2}{*}{$\begin{array}{l}\text { Tipos de contrações } \\
\text { avaliadas }\end{array}$} & \multicolumn{3}{|c|}{ sEMG * } & \multicolumn{3}{|c|}{$\mathrm{p}^{* *}$} \\
\hline & Dorsal & Sentada & Ortostática & $\mathrm{p} 1$ & p2 & p3 \\
\hline Repouso & $3,0(0-10)$ & $3,0(1-14)$ & $4,0(1-11)$ & 0,332 & 0,010 & 0,121 \\
\hline Fásica & $23,5(5-73)$ & $20,0(2-69)$ & $16,5(3-67)$ & 0,172 & $<0,001$ & 0,027 \\
\hline Tônica $10 \mathrm{~s}$ & $18,0(3-58)$ & $16,0(0-58)$ & $12,5(2-54)$ & 0,098 & $<0,001$ & 0,027 \\
\hline Tônica $20 \mathrm{~s}$ & $17,0(2-48)$ & $15,5(1-48)$ & $13,5(2-41)$ & 0,204 & 0,003 & 0,019 \\
\hline
\end{tabular}

*Valores em medianas (valores mínimos e máximos encontrados).

${ }^{* *}$ Teste de Wilcoxon para amostras pareadas.

$\mathrm{p} 1=$ dorsal $\mathrm{x}$ sentada; $\mathrm{p} 2=$ dorsal $\mathrm{x}$ ortostática; $\mathrm{p} 3=$ sentada $\mathrm{x}$ ortostática.

$10 \mathrm{~s}=10$ segundos; $20 \mathrm{~s}=20$ segundos.

\section{Discussão}

Os resultados do presente estudo demonstraram que a atividade eletromiográfica dos músculos do assoalho pélvico foi maior em decúbito dorsal e diminuiu progressivamente na posição sentada e ortostática. Quando se compararam as três posições, as contrações fásicas e tônicas na posição ortostática foram significativamente inferiores em relação ao decúbito dorsal e à posição sentada. Comparando somente os valores eletromiográficos da contração máxima do assoalho pélvico nestas mesmas posições, Laycock $^{3}$ também encontrou maior contratilidade em decúbito dorsal em relação à posição sentada e ortostática. Em contraste, Bo e Finckenhagen ${ }^{2}$ não encontraram diferenças entre as contrações em decúbito dorsal e na posição ortostática após o tratamento da IUE com cinesioterapia.

Embora os resultados do presente estudo se- jam provenientes de amostra de mulheres no menacme, Aukee et al. ${ }^{12}$ também observaram, entre as menopausadas, menor atividade muscular na posição ortostática em relação ao decúbito dorsal, e aquelas com IUE apresentaram contrações inferiores em ambas as posições. De acordo com Gunnarsson e Mattiasson ${ }^{6}$, o aumento da idade seria responsável por dano neuromuscular progressivo do assoalho pélvico e, conseqüentemente, diminuição da atividade eletromiográfica.

Como no presente estudo a avaliação eletromiográfica foi realizada somente após o tratamento, cujo objetivo era fortalecer os músculos do assoalho pélvico nas três posições, esperávamos que a capacidade de contração deste grupo muscular fosse semelhante nas três posições avaliadas. Entretanto, como não foi observada diferença entre as posições sentada e decúbito dorsal, supõe-se que na posição ortostática a ativação muscular seja realmente menos efetiva que nas demais posições. Uma explicação para este resultado é a ação 
mais intensa da gravidade exercida sobre as vísceras pélvicas e, conseqüentemente, sobre a musculatura do assoalho pélvico, na posição ortostática. Em situações de sobrecarga, o assoalho pélvico poderia diminuir sua capacidade de gerar contrações musculares satisfatórias, o que seria traduzido diretamente por menores amplitudes de sinais eletromiográficos e, indiretamente, por uma força muscular reduzida. Entretanto, Dietz e Clarke $^{4}$, avaliando funcionalmente o assoalho pélvico com a ultra-sonografia perineal, não encontraram nenhuma influência da postura na contratililidade destes músculos. Mesmo assim, muitas mulheres do referido estudo foram incapazes de contrair satisfatoriamente os músculos na posição ortostática ${ }^{4}$. Ainda que alguns estudos não encontrem diferenças significativas entre as posições, a maioria deles concorda que as contrações geradas pelo assoalho pélvico são menos intensas em ortostatismo ${ }^{2,3}$.

Já no repouso, as amplitudes médias foram superiores na posição ortostática, no presente estudo. Como o repouso reflete o tônus basal muscular, talvez essas amplitudes superiores na posição ortostática também tenham ocorrido em resposta à ação da gravidade e sejam provenientes da ativação de fibras tônicas em detrimento das fásicas, mais solicitadas durante as manobras de esforço. Amplitudes de repouso com valores semelhantes aos aqui observados têm sido encontradas por outros autores que também estudaram mulheres após o tratamento fisioterápico da IUE, sugerindo que estas mudanças são decorrentes da hipertrofia muscular ${ }^{13}$.

Baseados em nossa experiência clínica e também na de outros autores ${ }^{2,3,12,13}$, acreditamos que o decúbito dorsal continua sendo uma das posições mais fáceis para se realizar a avaliação do assoalho pélvico. Ainda é dificil conduzir uma avaliação fisioterápica na posição ortostática, pelo fato de consumir mais tempo, de não haver consenso em relação ao melhor equipamento e, sobretudo, por ser menos confortável para ambos, paciente e terapeuta. Entretanto, a avaliação na posição ortostática parece ser bastante válida para adequada caracterização da capacidade funcional do assoalho pélvico e do seu mecanismo de relação com a IUE.

Embora o grupo estudado tenha realizado os exercícios perineais nas três posições, os resultados das amplitudes das contrações foram inferiores na posição ortostática, sugerindo que o fortalecimento muscular deve ser intensificado nas posições em que há ação mais intensa da gravidade. Todavia, novos estudos devem ser conduzidos a esse respeito.

\section{Referências}

1. Peschers UM, Gingelmaier A, Jundt K, Leib B, Dimpfl T. Evaluation of pelvic floor muscle strength using four different techniques. Int Urogynecol J Pelvic Floor Dysfunct. 2001; 12:27-30.

2. Bo K, Finckenhagen HB. Is there any difference in measurement of pelvic floor muscle strength in supine and standing position? Acta Obstet Gynecol Scand. 2003; 82:1120-4.

3. Laycock J. Clinical evaluation of pelvic floor. In: Schussler B, Laycock J, Norton P, Stanton S, editors. Pelvic Floor Re-education. Principles and practice. New York: Springer; 1994. p. 42-8.

4. Dietz HP, Clarke B. The influence of posture on perineal ultrasound imaging parameters. Int Urogynecol J Pelvic Floor Dysfunct 2001; 12:104-6.

5. Koelbl H, Strassegger H, Riss PA, Gruber H. Morphologic and functional aspects of pelvic floor muscles in patients with pelvic relaxation and genuine stress incontinence. Obstet Gynecol. 1989; 74:789-95.

6. Gunnarsson M, Mattiasson A. Female stress, urge and mixed urinay incontinence are associated with a chronic and progressive pelvic floor/vaginal neuromuscular disorder: an investigation of 317 healthy and incontinent women using vaginal surface electromyography. Neurourol Urodyn. 1999; 18:613-21.

7. Glazer HI, Romanzi L, Polaneczky M. Pelvic floor muscle surface electromyography: reliability and clinical predictive validity. J Reprod Med. 1999; 44:779-82.

8. Kobata AS, Takano CC, Castro RA, Sartori MGF, Baracat EC, Girão MJBC. Diagnóstico da incontinência urinária: eletromiografia. In: Ribeiro RM, Rossi P, Pinotti JA, editores. Uroginecologia e Cirurgia Vaginal. São Paulo: Roca; 2001. p. 48-52.

9. Herrmann V, Palma PCR, Ricetto CLZ, Dambros M. Definições e interpretação do estudo urodinâmico. Femina. 2003; 31:111-5.

10.Burns PA, Pranikoff K, Nochajski TH, Hadley EC, Levy KJ, Ory MG. A comparison of effectiveness of biofeedback and pelvic muscle exercise treatment of stress incontinence in older community-dwelling women. J Gerontol. 1993; 48:M167-74.

11.Glazer HI, Jantos MJ, Hartmann EH, Swencionis C. Electromyographic comparisons of the pelvic floor in women with dysesthetic vulvodynia and asymptomatic women. J Reprod Med. 1998; 43:959-62.

12.Aukee P, Penttinen J, Airaksinen O. The effect of aging on the electromyographic of activity of pelvic floor muscles. A comparative study among stress incontinent patients and asymptomatic women. Maturitas. 2003; 44:253-7.

13.Griffin C, Dougherty MC, Yarandi H. Pelvic muscles during rest: responses to muscle exercise. Nurs Res. 1994; 43:164-7. 